\title{
La estrategia metodológica de una investigación centrada en las regulaciones sexo genéricas en la universidad: reflexividad, implicación y contramemoria ${ }^{1}$
}

\section{Rafael Blanco ${ }^{2}$}

Recepción: 25 de abril de 2014 / Aprobación: 7 de agosto de 2014

\section{Resumen}

El objetivo de este trabajo es presentar una revisión de la estrategia metodológica cualitativa de una investigación finalizada. Esta tuvo por objeto analizar las regulaciones de las identidades de género y sexualidad en la vida cotidiana universitaria desde el punto de vista de los y las estudiantes. La investigación revistió una serie de particularidades: la universidad constituyó el espacio social en el que se realizó la indagación a la vez que la institución desde la cual se realizó el trabajo; las regulaciones sexo genéricas fueron objeto de indagación desde una posición siempre genérica y sexuada; la distancia investigativa se combinó con la cercanía de los sujetos entrevistados, debido a la proximidad generacional y el universo de acontecimientos compartidos de la época. Para la revisión metodológica propuesta se recurre a los conceptos de reflexividad, implicación y contramemoria con el objeto de reflexionar en torno a dos puntos: los vínculos entre investigador y sujetos-objetos de investigación y acerca de los "usos" de la voz de las personas entrevistadas.

\section{Palabras clave}

Educación; reflexividad; implicación; contramemoria; género; sexualidad

\section{Abstract}

The aim of this piece is to revise the qualitative methodological strategy assumed in an already finished research project. The purpose was to analyze the regulation of gender and sexuality identities in everyday university life from the students' point of view. The project entailed a series of characteristics: the university was both the space studied and the space where the study was carried out; sex and gender regulations were researched from within a sexed and gendered position; given the age and shared

$1 \quad$ La investigación que dio origen a este artículo tuvo como resultado la tesis doctoral titulada Universidad, regulaciones sexo genéricas y vida cotidiana. La dimensión sexuada de la experiencia estudiantil, presentada en el año 2012 y dirigida por la doctora Sandra Carli.

2 Argentino. Doctor en Ciencias Sociales, Universidad de Buenos Aires (UBA), Argentina. Becario CONICET en el Instituto de Investigaciones Gino Germani y docente Regular de la Facultad de Ciencias Sociales, ambos en la UBA. Correo electrónico: rafaelblanco.fsoc@gmail.com 
experiences between the participants of the study, research distance was combined with proximity among them. In order to conduct the proposed methodological revision, the article makes use of concepts such as reflexivity, implication and counter-memory, with the aim of reflecting upon two aspects: the links between researcher and subjects-objects of study; and the uses made of the voices of the study participants.

\section{Keywords}

Education; reflexivity; implication; counter-memory; gender; sexuality

\section{Resumo}

O objetivo deste trabalho é apresentar uma revisão da estratégia metodológica qualitativa adotada em uma pesquisa já concluída. Pesquisa esta que analisou (ou, se você não quiser repetir pesquisa, pode ser também: "Nela foram analisadas") as regulações sociais das identidades de gênero e sexualidade presentes na vida cotidiana universitária desde o ponto de vista dos alunos. A pesquisa apresentou uma série de particularidades: a universidade que serviu de espaço social para as entrevistas é também a instituição pesquisadora; as regulações sexo genéricas foram objeto de análise a partir de uma posição genérica e sexuada; o distanciamento investigativo combinou-se a uma proximidade com os entrevistados, por conta da proximidade geracional e dos eventos de época compartilhados. Para a revisão aqui proposta tomou-se como base os conceitos de reflexividade, envolvimento e contra-memória, a fim de refletir sobre dois pontos: as relações entre pesquisador e sujeitos-objeto de pesquisa, e sobre o "uso" da voz dos entrevistados.

\section{Palavras chave}

Educação; reflexividade; envolvimento; contramemória; gênero; sexualidade

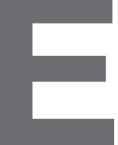

I objetivo de este trabajo es presentar una revisión de la estrategia metodológica cualitativa de una investigación ya finalizada. Esta tuvo por objeto analizar las regulaciones de las identidades de género y sexualidad en la vida cotidiana universitaria desde el punto de vista de los y las estudiantes. La investigación revistió una serie de particularidades: la universidad constituyó el espacio social en el que se realizó la indagación a la vez que la institución desde la cual se realizó el trabajo; las regulaciones sexo genéricas fueron objeto de indagación desde una posición siempre genérica y sexuada; la distancia investigativa se combinó con la cercanía con los sujetos entrevistados, debido a la proximidad generacional y el universo de acontecimientos epocales compartidos. Como sostiene Mario Pecheny, el carácter "políticamente pasional o apasionadamente político" que caracteriza a los estudios sobre géneros y sexualidades "la investigación sobre sujetos sexuales presenta varios desafíos" (2008, 9), especialmente, en torno a los vínculos entre investigador(a) y sujetos-objetos de investigación y acerca de 
los "usos" de la voz de las personas entrevistadas. Retomando estos desafíos, la reflexión de este artículo se centra en tres ejes: las decisiones tomadas en la delimitación del referente empírico, el cambio realizado en la estrategia de indagación durante el transcurso del trabajo de campo, y la elaboración de nuevas orientaciones en la indagación a partir de la reflexividad que envolvió el trabajo de entrevistas. En este análisis retomamos tres conceptos. Por un lado, "implicación" y "reflexividad" como categorías que permiten revisar y analizar el rol que juega quien investiga en el proceso de investigación. Por otro, el de "contramemoria" como categoría que permite aproximarse a las narrativas estudiantiles en tanto textos que propician una relectura de estas instituciones, invitando a revisar los alcances del carácter público de la universidad pública.

Para contextualizar el objetivo de esta indagación realizada entre 2007 y 2012 vale decir que la pregunta por las regulaciones sexo genéricas en esta institución estuvo motivada por los procesos de creciente visibilización y movilización en el espacio público de sujetos y colectivos de la diversidad sexual, de mujeres y feministas, producidos en la Ciudad de Buenos Aires entre la sanción de la Ley Porteña de Unión Civil, en 2002, y la Ley Nacional de Identidad de Género en 2012. Sin embargo, la afirmación de las y los sujetos de la diversidad sexual en escenarios cotidianos (lugares de trabajo, escuelas, espacios religiosos o familias, entre otros) en la Argentina contemporánea "constituye aún un camino por recorrer" (Moreno, 2008, 240) y es en esta dirección que la investigación se propuso interrogar qué sucedía, qué correlatos venían teniendo estos cambios, en una institución específica: la universidad pública. Así, se buscó responder, ¿qué procesos normativos orientan o prescriben las formas posibles de vivenciar la identidad y expresión de género y sexualidad, como varones, mujeres, trans, heterosexuales, lesbianas, gays, en la universidad pública? ¿Qué repertorios de normas, valoraciones, reglas de interacción, expectativas y modos de inteligibilidad regulan en la cotidianidad estudiantil? ¿Son distintos de los que operan "por fuera" de estas instituciones? ¿Es la universidad pública un escenario social "más plural" respecto de otros, según las narrativas estudiantiles? ¿Es más "conservadora", o no presenta diferencias? Para responder a estas preguntas se realizó un trabajo de investigación en dos facultades de la Universidad de Buenos Aires: en la de Psicología y en la de Ciencias Exactas y Naturales, indagando la presencia de estas regulaciones en la sociabilidad entre pares (los vínculos de amistades, noviazgos, grupos de afinidades y las fiestas estudiantiles y los momentos de ocio entre clases, entre otros acontecimientos) y en las experiencias y prácticas políticas (la militancia universitaria, la participación en grupos de diversidad sexual y de mujeres, las demandas en el cogobierno universitario, entre otras).

Los ejes señalados a ser abordados en este texto se encuentran relacionados por los dos nudos metodológicos mencionados y que, si bien se encuentran estrechamente vinculados al problema de investigación, pueden al mismo tiempo presentar elementos comunes a otros estudios que aborden el 
análisis de los procesos regulatorios de las identidades de género, sexualidad y la diversidad sexual desde las Ciencias Sociales.

\section{El problema de investigación: lo público, lo privado, lo íntimo}

La investigación adoptó una estrategia metodológica cualitativa, a partir de la perspectiva etnosociológica que combina distintos aportes. Siguiendo a Bertaux (2005), se realizó un trabajo comparativo identificando, a partir de recurrencias en los relatos, la detección de "itinerarios con trazos comunes" $(2005,105)$ en torno a las regulaciones sexo genéricas presentes en la experiencia universitaria de estudiantes de las dos facultades mencionadas. Para ello se trabajó sobre el análisis de 36 entrevistas no directivas y de las observaciones participante y no participante entre los años 2007 y 2011.

De acuerdo con Sandra Carli (2012), la pregunta por la experiencia estudiantil inicia una perspectiva que se propone analizar el presente de las universidades públicas "desde abajo", desde las voces de los sujetos anónimos, ya que en la vida cotidiana de estos intervienen procesos de identificación inter e intra-generacional y de filiación con la institución mediante los que se asumen, se apropian y cuestionan rasgos de las culturas institucionales de las universidades públicas. Estos procesos permiten atender tanto al modo en que estas instituciones operan en la producción de los y las estudiantes así como también a las formas en las cuales estas son producidas (renovadas, desestabilizadas o confirmadas) a partir de las múltiples tácticas y estrategias que despliegan sus actores. Es decir, esta perspectiva invita a ver no solo cómo los sujetos atraviesan las instituciones (cómo los y las estudiantes "pasan" por la universidad) sino también cómo estos son atravesados, con distintas intensidades, por la institución. En palabras de Carli, se trata de poner en suspenso las enunciaciones universalistas tan características del discurso universitario, inscribiendo en el debate cuestiones vinculadas con los itinerarios biográficos, la vida cotidiana de las instituciones y las experiencias sociales y culturales, como un modo de leer los procesos de construcción histórica de la universidad (Carli, 2012, 25).

En esta dirección, la investigación tuvo como horizonte de reflexión revisar los alcances de "lo público" de la universidad pública. Si en la tradición argentina la universidad carga con los sentidos de acceso irrestricto, gratuidad, pluralidad y masividad -o acceso universal ${ }^{3}$ - que configuran, en relación con lo público, su historia en el siglo pasado y su promesa de inclusión aún vigente, la atención al funcionamiento de las regulaciones sexo genéricas en la experiencia

3 Se considera modelo de acceso universal al que contempla al 35\% de la de población en edad correspondiente a participar en el nivel de educación superior; "de masas", al 15\%, y de elite, debajo del 15\%, siendo la tasa promedio de matrícula en América Latina del 17,4\% (García Guadilla, 2002). 
estudiantil permite reactivar otros sentidos: aquello accesible a la mirada, a los regímenes de visibilidad y a las alternativas posibles y el conjunto de opciones identitarias y expresivas habilitadas para encarnar el tránsito por la institución.

La descripción de estas regulaciones desde la perspectiva de uno de los actores constitutivos de la vida universitaria se realizó retomando aportes de varias disciplinas y campos de conocimiento, a partir de la reconstrucción de lo que una larga tradición de los enfoques etnográficos ha denominado la perspectiva del actor, el punto de vista de los nativos o el conocimiento local. Estas expresiones nombran de diversas maneras un constructo metodológico creado y orientado teóricamente por quien investiga, y que tiene por objeto "dar cuenta de la realidad empírica tal como es vivida y experimentada por los actores" (Guber, 1991, 71). Adopto la denominación perspectivas de los actores, en plural, para enfatizar la polifonía que configura la experiencia universitaria. El interés estuvo puesto en identificar en los relatos sobre la cotidianidad universitaria las formas como los y las estudiantes experimentan (vivencian, sienten, disfrutan, evalúan) su condición genérica y sexuada en tanto actores de la vida universitaria. Mediante la reconstrucción de estos puntos de vista busqué informar acerca de aquello no documentado que, como especifica Elsie Rockwell (2009), en los estudios contemporáneos refiere a lo familiar y cotidiano, a lo invisible e invisibilizado.

Durante el proceso de investigación y hasta una vez finalizado, distintos acontecimientos fueron evidenciando la progresiva problematización del espacio universitario en esta dirección propuesta. En el año 2009, en coincidencia con el período cuando se realizaba esta investigación, parte de la comunidad académica de la carrera de Sociología de la UBA junto con un colectivo de artistas realizaron una intervención artístico-política denominada Baño Revolution. Esta consistió en modificar los íconos que indican la identidad femenina o masculina de los baños de una de las sedes de la Facultad de Ciencias Sociales, remplazándolos "por una multiplicidad de coloridas siluetas" diferentes de las conocidas, con el propósito de desnaturalizar "la binariedad impuesta", y buscando así transformar "Ios baños en espacios de una universalidad que pretendía incluir todos los cuerpos y todos los géneros" en la Facultad (Aguilar et al., 2009, 20). En 2012, por decisión de las autoridades de la Facultad de Periodismo y Comunicación de la Universidad Nacional de La Plata (UNLP), dejaron de existir los baños separados por géneros: el comunicado institucional señala que "a partir de hoy, lunes 4 de junio, no existirán baños separados para varones y mujeres, sino que ahora serán en forma indistinta para varones, mujeres y para lo que cada uno decida y quiera ser"4. Ambos hechos dieron lugar a una intensa polémica periodística que se desarrolló en distintos registros desde el debate de ideas al agravio en torno a la naturalización de

4 Facultad de Periodismo y Comunicación (UNLP). Recuperado de http://www.perio.unlp.edu.ar/ node/2182 
las relaciones sociales y las tensiones entre lo que es público, lo privado y lo íntimo en la vida universitaria. ¿Cómo conjugar estas tensiones durante la indagación, en la interacción con los sujetos? ¿Cómo articularlas en la instancia de la entrevista, esa "escena fundante", al decir de Leonor Arfuch (1992), de la investigación cualitativa?

\section{La entrevista y los sujetos entrevistados: indicios y experiencias en situación}

La relación entre universidad y regulaciones sexo genéricas permite ser abordada a partir de múltiples entradas analíticas: currículos, programas y formatos institucionales (cátedras, institutos, grupos de estudio y materias electivas, entre otros) que abordan el género y las sexualidades como campo de conocimiento en el ámbito académico. Las normativas específicas que regulan las expresiones e identidades de género (como las que se instauraron en distintas casas de estudio con anterioridad a la sanción de la Ley de Identidad de Género, que validan el nombre autopercibido para la realización de trámites como la identificación en padrones electorales, listas, constancias de asistencia); las políticas gubernamentales o las de las propias universidades que han tomado como destinataria a la población universitaria (por ejemplo, en materia de salud sexual), y las representaciones y prácticas sexuales de tal o cual actor de la vida universitaria (estudiantes, docentes, trabajadores), entre otras posibilidades. No obstante, vínculo entre universidad, vida cotidiana, género y sexualidad fue abordado considerando el análisis de la experiencia universitaria de estudiantes en las facultades de Psicología y de Ciencias Exactas y Naturales. La elección de ambas instituciones es el resultado, en un plano, de un trabajo exploratorio preliminar realizado en siete instancias de la UBA. En otro -y en relación con el primero- de la estrategia analítica que se orientó a establecer puntos de comparación entre unidades académicas.

El trabajo de entrevistas y observación se dividió en dos etapas: una primera aproximación a un conjunto mayor de unidades académicas entre mayo y noviembre de 2008 y, luego, el trabajo de campo propiamente dicho (es decir, la delimitación de coordenadas espacio temporales de indagación), que tuvo lugar entre marzo de 2009 y noviembre de 2011. Este recorte temporal coincide con la mayor presencia en la agenda pública (legislativa y mediática) debido a las discusiones iniciadas en 2002 en torno al matrimonio entre personas del mismo sexo, la identidad de género, la trata de personas o la violencia de género, en el que se produjeron regulaciones jurídicas específicas, y que transcurrida más de una década, invitan a interrogar en el presente los alcances de estas transformaciones en los ámbitos cotidianos. Como fue dicho, la elección de las facultades de Psicología y Ciencias Exactas se relaciona con la posibilidad de establecer comparaciones entre modos de atravesar la vida universitaria en instituciones con características diferentes y constituye, a su vez, el emergente 
de una situación de asombro -la "apertura a la sorpresa" como la nombra Rockwell (2009) - durante el inicio del trabajo de entrevistas y observación.

La primera etapa de la indagación fue realizada en las facultades de Ciencias Económicas, Medicina, Derecho, Arquitectura, Diseño y Urbanismo, además de en Exactas y Naturales, y en Psicología. La exploración en este conjunto de facultades buscó atender a instituciones con identidades, perfiles e historias diversas: de perfil profesional o científico, de la etapa fundacional de la universidad o surgidas durante la segunda mitad de siglo XX, emplazadas en la trama urbana o ubicadas en el campus espacialmente construido de la Ciudad Universitaria, que tuvieran -o no- en sus currículos contenidos relativos al género, al sexo o a la sexualidad. Con el fin de lograr un mayor distanciamiento con el universo empírico de análisis y teniendo en cuenta lo que en el siguiente apartado se caracteriza como la particularidad de una investigación desarrollada en un "universo próximo", se excluyeron de este trabajo exploratorio las facultades de Ciencias Sociales y de Filosofía y Letras en las que había desarrollado mi propia formación de grado. Un supuesto en esta búsqueda -y que se constituyó como hipótesis estructurante de la investigación - fue que las modalidades de regulación de expresiones e identidades de género y sexualidad en el espacio universitario se producen en estrecha relación con las particularidades institucionales de cada Facultad y los repertorios culturales disponibles en cada una de ellas.

De las primeras observaciones registradas en las notas de campo surgieron algunos indicios: las rutinarias inspecciones de los baños de varones que realiza varias veces al día el personal de seguridad (identificado así por la leyenda impresa en sus abrigos) de la Facultad de Ciencias Económicas, ¿constituían un signo de control sobre un espacio que habitualmente es resignificado como escenario de actividad sexual? (Humphreys, 1975). La convocatoria de la Comisión de Mujeres de la Facultad de Arquitectura, Diseño y Urbanismo (FADU) al "XIII Encuentro Nacional de Mujeres" realizada en un enorme cartel que se desplegaba hacia el patio central con el rostro de Mao Tse Tung y una frase del líder chino ("Las mujeres llevan sobre sus espaldas la mitad del cielo y deben conquistarlo"), ¿podía leerse como un indicio de la emergente "reconversión al género" de la agenda de las agrupaciones estudiantiles? El comentario de un estudiante que militaba en una agrupación universitaria y, en paralelo, en un espacio de diversidad sexual por fuera de la Facultad (sin que sus compañeros de militancia universitaria supieran de su activismo: "después cada uno seguía con su vida", en palabras del entrevistado), ¿indicaba el carácter heteronormativo del espacio universitario, la necesidad para algunos y algunas de desplegar "identidades discretas" (Pecheny, 2002), sino secretas?

Numerosas impresiones y vicisitudes registradas en estas notas fueron retomadas de cara al trabajo de observación participante y de entrevistas de la segunda etapa. Devinieron así bisagra entre el campo empírico y la elaboración teórica, a partir de las anotaciones que, a la manera de un "diálogo de 
la perplejidad" (GTTCE, 1999, 71), orientaron posteriormente la indagación y la escritura. Proceder desde indicios permitió ir vinculando aquellos hechos triviales o extraordinarios que resultaban significativos en conjunto con sus localizaciones espaciales y temporales precisas. Como recuerda Aníbal Ford (1996), el índice es un signo que se decodifica contextualmente, y atender a ellos permite generar hipótesis sobre comportamientos culturales en situación. En este sentido, la elección de Ciencias Exactas y Naturales, y Psicología se relaciona con el asombro (un tipo de índice) ante dos acontecimientos que, por su particularidad o su redundancia, se recortaba del flujo cotidiano en estas facultades.

En Exactas, durante el inicio del trabajo de indagación comenzaban a realizarse las primeras reuniones públicas de un grupo de diversidad sexual. En hojas tamaño A4, blanco y negro, pegadas en distintos espacios de circulación del edificio (pasillos, descansos de escalera, baños) podía leerse: "Ciclo de cine 'Ensalada Cerebral' y CHEN (Comunidad homosexual de exactas y naturales). Proyectaremos Shortbus en el contexto de cine LGBT (lésbico, gay, bisexual, trans). Viernes 12/09, 20 hs Aula Magna del Pabellón II”5. En el paisaje habitual de esta Facultad, dominado por grandes letreros que tematizaban la insuficiencia del presupuesto universitario, el rol de la universidad en la sociedad o diversos conflictos con el Estado nacional ("¿Qué esperamos de la Educación Superior? Educación para el cambio social”, "¿Redistribución de la riqueza? ¡Basta de Verso!", "Anulación ya de la Ley de Educación Superior", "Ciclo de charlas Economía y Ciencia", entre otros), que interpelan a partir de identidades políticas reconocibles por su tradición en la universidad ("Izquierda socialista", "Nueva Izquierda", "ConCiencia Crítica", "Unión de Juventudes por el Socialismo"), la proyección en el aula magna de la Facultad de un filme con recurrentes escenas de sexo explícito que transcurren en un club nocturno de Nueva York (llamado Shortbus), parecía establecer cierta disrupción.

En cuanto a Psicología, la sorpresa estuvo dada por la presencia de una acción sostenida por parte de las agrupaciones estudiantiles de realización de eventos en torno a "la mujer" y "el género", como la "charla debate: crisis capitalista, trata y violencia hacia la mujer" organizada por la Presidencia del Centro de Estudiantes en el marco de la "Jornada contra la trata" e impulsada por la comisión de mujeres de la Federación Universitaria de Buenos Aires (FUBA); el ícono de una mujer embarazada con un libro bajo el brazo con la leyenda "Dale el sí a la cursada para embarazadas de la Secretaría de Políticas de Género" pegado en diferentes espacios comunes de la Facultad; otro afiche impreso a colores que, sobre un pizarrón, anunciaba la "Campaña latinoamericana por el derecho al aborto legal, libre, seguro y gratuito", firmado por la agrupación "Pan \& Rosas", o uno repetido nueve veces en el descanso de una escalera que

5 Registro de observación realizado el 10 de septiembre de 2008 en la Facultad de Ciencias Exactas y Naturales. 
conecta el patio central con el primer piso que invitaba: "28 de mayo. Día Internacional para la Salud Integral de la Mujer. Video-taller por el derecho al aborto. Hall de Independencia, 19:30 hrs"6. Lo disruptivo se relacionaba con la recurrencia temática de "género" y "mujer" por contraste con las otras facultades.

Por su singularidad o su repetición, ambos fenómenos permitieron avanzar desde la observación y el registro a la interrogación: ¿por qué allí? (¿Por qué un grupo de diversidad sexual en Ciencias Exactas y Naturales?, ¿por qué la problematización respecto del género en una Facultad que tematiza fuertemente la sexualidad, como es la de Psicología?). Como primeros indicios, estos dos acontecimientos (de un grupo de diversidad sexual; distintos grupos abocados a cuestiones de género) resultaron de interés por su carácter excepcional: constituían una marca distintiva en relación con el resto de las unidades académicas en las que se realizaba el trabajo exploratorio, a la vez que una seña particular de cada una de estas instituciones, que invitaba a prestar atención a sus particularidades.

Sin embargo, el procedimiento de indagación no se orientó hacia la descripción particularizada de estos fenómenos, sino de los vínculos de estos con el entramado más amplio en el que se inscriben y del que adquieren su sentido, siguiendo la pista de Giorgio Agamben (2003) respecto de las relaciones estrechas entre norma y excepción. Es decir, la investigación se orientó a indagar estos dos fenómenos no en sus características intrínsecas, sino a partir de sus relaciones con "la normalidad" de la vida cotidiana: si esta es el terreno de la construcción de sentidos que orientan nuestra sociabilidad (Berger y Luckmann, 2006; Lindón, 2000), ¿qué sentidos son producidos en relación con las expresiones e identidades de género y sexualidad en el espacio universitario?, ¿cómo se materializan en las prácticas cotidianas de los y las estudiantes de Psicología y Ciencias Exactas y Naturales? En definitiva: ¿qué sociabilidades producen?

El pensamiento de la excepcionalidad de Agamben (2003), si bien toma otros objetos de reflexión, permitió articular los dos acontecimientos descritos (en torno a la diversidad sexual en Exactas y al género en Psicología) con su contexto cotidiano de emergencia. La pregunta respecto del vínculo entre los fenómenos referidos como "sorpresivos" en cada una de las facultades y el entramado institucional donde tienen lugar, dio paso a la emergencia del supuesto que orientó la indagación en terreno: que la normatividad sexo genérica se produce en y por una serie de características particulares de estas facultades, características que intervienen en la modulación de los itinerarios estudiantiles en función de los repertorios culturales disponibles en cada una. En otras palabras, que el modo como las expresiones e identidades de género y sexualidad son posibles para los y las estudiantes en el espacio universitario varía en cada Facultad en función de las formas en las cuales se desenvuelve la experiencia estudiantil en cada institución. Por ello, las entrevistas estuvieron

6 Registro realizado el 4 de junio de 2009 en la sede Yrigoyen de la Facultad de Psicología. 
orientadas a reconstruir la vida cotidiana, los acontecimientos ordinarios, las interacciones habituales, de los y las estudiantes en sus facultades.

Las entrevistas realizadas fueron de carácter no directivo: si bien estuvieron dirigidas a recabar información previamente definida por los propósitos de la investigación -y plasmada en una guía de entrevista-, fueron abiertas a que las respuestas se convirtieran en nuevas preguntas no estipuladas previamente. Como fue dicho y en pos de reconstruir tanto los trazos generales de la experiencia universitaria como la singularidad de este tipo de regulaciones, la guía estaba orientada a que las personas entrevistadas narrasen y describieran los rasgos habituales que adquiere la vida universitaria: las primeras impresiones de la Facultad, la conformación de grupos de amistades, militancia y estudio, las visiones sobre los pares y sobre la institución (reconstruyendo situaciones como las clases, las fiestas, las asambleas estudiantiles, los exámenes), el relato de los distintos lazos establecidos (de amistad, de noviazgo y de "cortejo", entre otros), las visiones e impresiones sobre la política estudiantil y la relación con el conocimiento (los vínculos entre los temas de interés y la vida cotidiana, la perspectiva laboral y profesional, las materias que han resultado significativas, la elección de materias optativas o la complementación con espacios y grupos de formación extracurriculares), otros aspectos previos al inicio de la vida universitaria (estudios anteriores, grupos de amigos, viajes, entre otros hechos significativos) como así también las características familiares y laborales.

Estas orientaciones procuraron recoger las descripciones de acontecimientos cotidianos presentes en aquellos discursos informales que emergen constantemente en la vida diaria (comentarios, anécdotas, recuerdos de conversaciones o situaciones) (Guber, 2001) y en los que se plasman significados referidos a las expresiones e identidades de género y sexualidad en la universidad. Se buscó así registrar "relatos de prácticas en situación" (Bertaux, 2005) como forma de comprender de manera más cabal los contextos sociales que pertenecen a sus condiciones de producción. La modalidad no directiva posibilitó una reflexividad de los sujetos entrevistados sobre su propia biografía ("a mí me viene bien este espacio, me hace pensar", comenta una estudiante de Psicología respecto de la entrevista) al mismo tiempo que habilitó la interpelación de estos al investigador (“¿Tu interés tiene que ver con tu atravesamiento por la Facultad de Sociales?", como me fue preguntado por una entrevistada de Psicología respecto de mi propia experiencia).

Las entrevistas fueron realizadas en los lugares concertados con las y los entrevistados. Sin embargo, hubo una marcada regularidad: mientras que los encuentros con estudiantes de Exactas se desarrollaron mayormente en espacios de la Facultad (el comedor de alguno de los pabellones; también, en escalinatas o en la biblioteca), en el caso de quienes estudian en Psicología, se concretaron, o bien, en bares y cafés aledaños a la Facultad o cerca de los lugares de trabajo. La posibilidad de realizar los encuentros en el edificio de 
interrogar los vínculos entre la cotidianidad en ese espacio y las formas en que son reguladas las expresiones e identidades de género y sexualidad en una facultad en la que género y sexualidad -a diferencia de Psicología, debido a su tradición psicoanalítica- no constituyen centralmente objeto de conocimiento, por lo que su puesta en discurso en la escena pública es escasa, o bien se desarrolla por canales extra curriculares. Por caso, en una entrevista realizada en el comedor del Pabellón I, sobre la pared contigua a la mesa en la que nos encontrábamos entrevistado y entrevistador, se hallaba pegado un cartel en hoja A4 con la leyenda que sigue: "1/11- 18 hrs- Plaza de Mayo. Desde la CHEN invitamos a toda la comunidad de la FCEyN a participar de la 18va marcha del orgullo LGTB (Lésbico, Gay, Trans, Bisexual)". Esto dio pie a la pregunta por los vínculos entre la visión del entrevistado sobre su Facultad (a la que había calificado como un ambiente "progre" [liberal]) y el surgimiento de una agrupación de diversidad sexual en la Facultad ("cuando decías que era una Facultad progre, recién veía el cartel convocando a la 'marcha del orgullo', ¿Esa sería una marca del carácter progre?”).

Las personas entrevistadas fueron contactadas durante el trabajo de observación participante en cada Facultad y a partir del mecanismo de "bola de nieve" (Bertaux, 2005, 60), se consultó a cada sujeto entrevistado si sabía de pares que pudiesen estar interesados en participar. Se realizaron 36 entrevistas a estudiantes avanzados de las carreras de Psicología (Facultad de Psicología) y de Biología, Física, Química y Ciencias de la Computación (Facultad de Ciencias Exactas y Naturales), muchos de los cuales se encontraban cursando las últimas materias, lo que posibilitó la recuperación retrospectiva de su paso por la universidad procesada por la experiencia posterior y por las expectativas e interpretaciones de la situación de entrevista (Sautu, 2004). El número y las características de las personas entrevistadas fue definido por criterios de saturación teórica (Glaser y Strauss, 1967), es decir, el trabajo finalizó cuando no se logró hallar ninguna nueva "información" relevante a las ya proporcionadas en otras entrevistas.

Los sujetos entrevistados de ambas facultades cursaron y aprobaron el Ciclo Básico Común -el ciclo de materias de ingreso a la UBA- entre los años 2002 y 2005, y comenzaron su carrera entre los años 2003 y 2006. Cuando se llevó a cabo la entrevista, tenían entre 23 y 29 años. Entre quienes estudian en Psicología, una minoría comenzó en paralelo o con anterioridad otra carrera universitaria. La mayoría realizaba sus estudios hasta el momento de la investigación trabajando o había trabajado la mayor parte del tiempo de cursada. En general, se desempeñaban o se habían desempeñado en empleos que no requieren título universitario ni conocimientos ligados a su formación. Salvo excepciones, los trabajos realizados no se encontraban relacionados con la formación disciplinar y profesional. En su mayoría, las personas entrevistadas viven en la Ciudad de Buenos Aires, aunque una alta proporción de estudiantes reside en localidades de los cordones urbanos oeste y sur 
del Gran Buenos Aires. Una baja proporción se desplazó de otra provincia para realizar sus estudios en la UBA, decisión en la que pesó fuertemente el prestigio familiar otorgado a esta institución. Fenómeno poco habitual en Ciencias Exactas y Naturales, una minoría de quienes fueron entrevistados son la primera generación de universitarios de su familia. En proporciones similares, proceden de colegios de gestión estatal y de gestión privada, muchos de ellos religiosos. En Exactas, por su parte, la mayoría de las personas entrevistadas proviene de colegios estatales, y una alta proporción, de instituciones medias dependientes de la UBA (el Colegio Nacional Buenos Aires o la Escuela Superior de Comercio Carlos Pellegrini). En su mayoría viven en la Ciudad Autónoma de Buenos Aires, y una minoría en localidades del sur bonaerense. A diferencia de quienes cursan en Psicología, los y las estudiantes de Exactas consiguen trabajos relacionados con su formación, según la disciplina. En una baja proporción desarrollan tareas administrativas en el Estado o empresas privadas?.

Los perfiles de los sujetos entrevistados fueron variando a medida que avanzaba la investigación. Participaron en diferentes instancias del trabajo de entrevistas tanto estudiantes que militaban en alguna agrupación estudiantil así como también otros que no realizaban actividad alguna en este sentido o que habían tenido alguna participación coyuntural. En concordancia con la conformación de las características estudiantiles, la proporción de estudiantes mujeres entrevistadas fue mayor en Psicología, mientras que en FCEyN la cantidad de entrevistas realizadas a varones y mujeres fue equivalente. Por último, en el transcurso de las entrevistas, una minoría de estudiantes varones de ambas facultades se refirió a sí mismo como gay ("el último verano me asumí gay", "soy gay"), otros y otras asumieron implícitamente una identidad heterosexual ("estaba saliendo con una chica de acá de la Facultad", como narra un entrevistado, o una estudiante de Psicología que interrumpe la entrevista para decir: "Perdoname, ¿ves el chico que está ahí, que está ahí afuera? Es hermoso. Esas cosas que aparecen ahora que ya estoy casada"). Nadie asumió, tácita o manifiestamente, otra identidad de género o de sexualidad "por fuera" de la división varón/mujer y heterosexualidad/homosexualidad masculina. Esto último constituyó otro indicio respecto de las regulaciones de las expresiones de género y sexualidad en el espacio universitario, lo que permitió elaborar una nueva hipótesis de investigación: que a diferencia de las mujeres lesbianas, poco visibles en el espacio de la Facultad y de las personas trans, la homosexualidad masculina es objeto de referencia habitual para caracterizar a los actores de la vida estudiantil así como también una forma de identificación disponible en el repertorio cultural de las facultades analizadas. 


\section{Implicancia, reflexividad y el cambio de estrategia de indagación}

El trabajo sobre las perspectivas de los actores no implicaron recoger un testimonio transparente y centrado en la voluntad de un yo -"la ilusión biográfica" como denomina Bourdieu (1997) a esta mirada-, sino concretar una construcción mediada por la investigación y realizada a partir de una multiplicidad de registros: las entrevistas no directivas, la observación participante y las notas de campo que permiten inscribirlas en procesos sociales más amplios. Esta construcción suscitó un problema central, sobre el que anudó la estrategia metodológica: el trabajo de análisis de lo que fue mencionado en el inicio del artículo como un universo próximo.

La proximidad refiere a lo que René Lourau (1991) denomina implicación, al nudo de relaciones (las adhesiones y motivaciones y sus contrarios, al conjunto de referencias puestas en juego) siempre presente en el trabajo de investigación, que debe ser analizado de modo tal que la subjetividad de quien investiga no se convierta en una caja negra. Como fue mencionado al inicio, la universidad representa un universo próximo en el sentido de que esta institución es constitutiva del objeto de investigación a la vez que el espacio institucional desde el cual realicé la investigación; la experiencia universitaria, una dimensión que se pretende reconstruir y sobre la cual se va a indagar a la vez que es un rasgo de la propia biografía del investigador; las regulaciones sexo genéricas, objeto de descripción desde una posición siempre genérica y sexuada. Por último, el problema planteado implica una cercanía relativa con los sujetos entrevistados, dada por la proximidad generacional y un universo de acontecimientos compartidos y significativos entre estos y quien investiga ${ }^{8}$.

La implicación operó tanto en la instancia de realización de las entrevistas como en la de análisis y escritura. En el desarrollo de las entrevistas, algunas de estas cercanías constituyeron tanto un elemento facilitador (por la posibilidad de entablar en intercambio mediado por un lenguaje común) como así también un aspecto que dificultó en determinados momentos la profundización en algunos temas emergentes durante el intercambio. La facilidad estaba dada por esa experiencia próxima, al decir de Clifford Geertz, que supone poseer un universo relativamente compartido de términos utilizados por un informante para definir lo que este o sus pares expresan de modo espontáneo, natural o coloquial. Para este autor, "un concepto de experiencia distante es, en cambio, aquel que los especialistas de un género u otro -un analista, un experimentalista, un etnógrafo, incluso un sacerdote o un ideólogo- emplean

8 "Generación" señala una serie de rasgos culturales e históricos que permiten recortar del conjunto de la sociedad a un grupo socializado bajo características comunes. Siguiendo a Carles Feixa, lo que diferencia a una generación de otra es, por un lado, una serie de factores históricos y estructurales y, por el otro, un sentimiento de "contemporaneidad" expresado por recuerdos en común, "acontecimientos de generación”, espacios comunes y auto calificaciones (Feixa, 1998, 52). 
para impulsar sus propósitos científicos, filosóficos o prácticos"; así, "el 'amor' es un concepto de experiencia próxima, mientras la 'catexis objetual' lo es de experiencia distante" (Geertz, 1994, 75). Por caso, cuando un entrevistado de Psicología comenta que cursar en una Facultad en la que la mayoría del estudiantado está compuesto por mujeres es "interesante", ya que brinda la posibilidad de "pegar veinte minas", la entrevista sigue su curso sin aparente necesidad de explicar a qué se refiere: constituyen enunciados inteligibles por una experiencia cercana, un lenguaje familiar.

Pero en esta misma cuestión anida una dificultad: los significados compartidos no necesariamente poseen los mismos referentes, que obedecen más bien a las distintas situaciones: ¿qué prácticas involucra "pegar"?, ¿cómo se "pegan veinte minas" en Psicología? Por otro lado, "mina", ¿refiere a un tipo específico de mujer?, y si es así, ¿qué características tiene? Por último, ¿qué secuencias de acontecimientos recrean la situación de "pegar una mina" en esta Facultad? Una relectura de la transcripción de la entrevista permitió advertir que si, por ejemplo, se hubiese profundizado en el significado de esa frase, habría sido posible indagar con mayor precisión, durante la entrevista, la sociabilidad afectiva y sexual habilitada en dicha unidad académica.

Este juego de "implicación-reflexividad", como denomina Valeria Hernández al proceso por el cual quien investiga revisa y analiza su implicación metódicamente "con el fin de hacer explícito su rol, de convertirlo en una fuente de conocimiento sobre las relaciones en las que ha participado" (Hernández, $2006,60)$ constituyó entonces una dimensión significativa para analizar la cotidianidad en ambas facultades y para realizar nuevas preguntas y conceptualizaciones a medida que avanzaba el trabajo de indagación. Así por ejemplo, si en Psicología la entrada, circulación o salida, o las conversaciones informales, revestían un alto grado de "libertad" durante el trabajo de observación participante (nadie parecía advertir en esta Facultad la actividad que realizaba como significativa), en Exactas la sensación fue siempre la de ser un alguien "foráneo" (impresión confirmada más tarde por algunos entrevistados que habían comentado entre sí mi presencia e intercambiado pareceres en cuanto a las entrevistas que realizaba o las conversaciones informales que mantenía).

A diferencia del modo de ingreso en Psicología, las primeras observaciones en Ciencias Exactas y Naturales -las primeras derivas, los recorridos por esta Facultad sin dirección fija- fueron en compañía de un informante clave. El alto grado de conocimiento entre sí de quienes concurren a la FCEyN, debido a las características de la vida en común alli ${ }^{9}$, obligó a un cambio en la estrategia

$9 \quad$ Los y las estudiantes permanecen allí un promedio de siete horas diarias. Esto se debe no solo a las horas lectivas que demanda cada materia, sino también a la existencia de espacios comunes de encuentro y permanencia: la biblioteca con su sala parlante y su sala silenciosa, el comedor central ubicado frente al río, que también constituye el paisaje del patio central y los balcones con mesas y sillas. En Psicología, no solo los cursos requieren una menor carga horaria, lo que prescribe una 
de indagación en esta unidad académica y en la investigación en general. A su vez constituyó un indicio de las formas de sociabilidad que tienen lugar allí (el tipo de lazo social predominante en cada Facultad) y su impacto en los procesos de regulación de las expresiones e identidades de género y sexualidad, especialmente, en torno a cómo la mirada y los rumores van modulando las experiencias personales.

Si en un primer momento, y debido a los lazos poco visibles entre universidad y regulaciones sexo genéricas, adopté una estrategia naturalista -la búsqueda de "pasar desapercibido"-, posteriormente abandoné esta modalidad. En el contexto de una institución en la que existe un alto grado de interconexión entre estudiantes, el intento por lograr "una fusión del investigador con los sujetos de estudio" que niega la propia implicación (Guber, 2001, 42) y que pretende describir las subjetividades de otros recurriendo a unas "supuestas capacidades 'más que normales' que nos permitirían tanto pasar inadvertido como generar un clima cordial de camaradería" (Geertz, 1994, 90), resultó inadecuada. Es por ello que la reflexividad sobre la indagación en Exactas aportaba otro dato: el de la sensación de ser un extranjero esforzándose infructuosamente por hablar una lengua que no le es propia.

El cambio de estrategia se centró entonces en la intensificación del control del propio comportamiento en un universo próximo con el objeto de lograr una distancia relativa con el universo de sentidos de los procesos que iba analizando (Frederic, 1998). El cambio tuvo que ver con explicitar desde el inicio el rol de investigador, algunos objetivos de la investigación, el marco institucional en el que esta se desarrollaba, e incluso, respondiendo aquello que era preguntado durante las entrevistas o en conversaciones informales como, por ejemplo, mi vinculación con la política, la militancia en la universidad y mi propia experiencia estudiantil e incluso mi identificación sexual. Esto redundó en una actividad constante, tensa y configurativa del trabajo de indagación que permitió la elaboración de nuevas preguntas.

Algunas tensiones registradas durante el transcurso del trabajo de entrevistas permiten ilustrar el cambio de estrategia y la emergencia de algunas nuevas orientaciones que fue adquiriendo la indagación. En una primera etapa, el modo inicial de comenzar la comunicación y de explicar el objetivo de la investigación fue mencionando que se trataba de describir las regulaciones del género en la universidad (en los primeros encuentros enuncié de modo recurrente: "busco dar cuenta de las normas de género en la Facultad según la visión de los estudiantes"). Esta entrada fue "exitosa": inteligible para quien era entrevistado, tranquilizadora para el entrevistador. La noción de género

presencia de tiempo parcial, sino que también las posibilidades de permanecer en el edificio son menores por la casi inexistencia de espacios comunes. Según el Censo de Estudiantes 2004 realizado por la UBA, en la FCEyN cursan 8.263 estudiantes (de los cuales el 73,1\% cursa en las carreras y el 26,9\% restante en el CBC). En Psicología el número se triplica: de los 26.054 estudiantes, el 71,6\% cursa en Facultad y el $28,4 \%$ restante en el CBC. 
se encuentra legitimada en la academia argentina: es hoy un tópico objeto de investigaciones y viene siendo retomado por el activismo estudiantil; estos acontecimientos, entre otros, marcan el carácter amplio del uso de este término. Por lo tanto, plantear que se trataba de una investigación que indaga "cómo el género juega" en la experiencia universitaria (aún sin explicitar qué se entiende por "género" en el marco de esta investigación: cada quien parece tener una representación más o menos compartida de lo que esto podría llegar a significar) constituyó un terreno de mayor comodidad para presentar el tema a las y los entrevistados. "Género" puede ser nombrado sin que eso presuponga una sobreimplicación -retomando la desviación utilitarista de la noción de implicación comentada críticamente por Lourau (1991)-, el grado de activismo, la identificación o carga afectiva excesiva que conllevaría una determinada tarea. La consagración del género en el discurso universitario y en el uso público permite a quien utiliza esta categoría cierto resguardo, cierto "lugar a salvo".

Esta estrategia fue parcialmente beneficiosa: al mismo tiempo que permitía iniciar un diálogo, implicaba un límite en la conversación y marcaba dificultades para abordar aspectos de las biografías de las y los estudiantes que se presentaban como relevantes en las entrevistas, pero que si se avanzaba sobre ello, parecía transgredir una pauta previa. Por ejemplo, cuando pregunté a un estudiante, quien había mencionado en algunas ocasiones durante la entrevista a un ex novio, si lo había conocido en los cursos, me respondió: "¿Tenemos que hablar de estas cosas? No, no salí con gente de la Facultad". Como enfatiza Leonor Arfuch, "por más científica que sea la mirada, [la entrevista] es siempre una intrusión en la privacidad" (Arfuch, 1992, 73), intrusión que, tratándose de un terreno que bordea la intimidad, requería delimitar previamente sus fronteras.

Por ello, el segundo punto de la estrategia de salida al campo fue intentar modos combinados de presentar el trabajo, haciendo referencia a que el objetivo era la descripción de las regulaciones sexo genéricas, las normas de género y sexualidad, el modo en que el género y la sexualidad son regulados en la experiencia universitaria, con el propósito de lograr un degradé de lo legítimo a ese otro plano más problemático que es el de nombrar la sexualidad en una institución "transparente"; retomando la figura utilizada en el inicio del texto, el vínculo entre sexualidad y universidad pareciera requerir explicación, y esta dificultad en la propia constitución del objeto de indagación deviene un problema pero a la vez en un dato mismo de la investigación.

Esta dificultad permitió ir elaborando una nueva hipótesis: para analizar las normas sexo genéricas en la universidad es necesario atender a la vergüenza que produce el tema de la sexualidad en este espacio e ir ubicando el modo en que esta modula las interacciones cotidianas, ya que este sentimiento opera como bisagra entre la experiencia subjetiva y aquellos con quienes uno, de diversos modos, se encuentra unido. La vergüenza, en efecto, resulta una 
modalidad regulatoria en la medida en que se traduce en acciones cotidianas que estructuran los vínculos intersubjetivos, como el miedo a la mirada, la prudencia o el "llamarse a silencio" frente a la opinión ajena (Elías, 2009), y fue por lo tanto un analizador relevante para comprender el funcionamiento de las regulaciones sexo genéricas en el espacio universitario.

Con sus particularidades según los escenarios culturales concretos (en este caso, las diferencias entre Psicología y Exactas), es el hecho de sentir vergüenza una de las principales modalidades de regulación de las expresiones e identidades de género y sexualidad, lo que explica -por caso- los silencios, la discreción y la autocensura, que marca la continuidad y no la distinción entre una esfera pública (no emocional) y una privada cargada de emociones (lllouz, 2007). Es la vergüenza, indicio surgido en el trabajo de campo, lo que condiciona y posibilita -"tanto se deriva como se dirige", en palabras de Eve Kosofsky-Sedgwick $(1999,203)$ - la sociabilidad. En contraste con las instituciones de educación primaria o secundaria en las que prácticas como el hostigamiento van produciendo las modalidades regulatorias en torno a géneros y sexualidades, en la vida universitaria no se advierte en las entrevistas y observaciones realizadas el funcionamiento de los mismos mecanismos normativos (como el acoso escolar o bullying) por más que sea necesario atender a su perdurabilidad aún después del fin del ciclo escolar (como afirma un entrevistado al narrar su llegada a la universidad: el hostigamiento en los otros niveles de formación "son marcas que en el colegio se rigidizan y perduran, quedan ahí"). Contrariamente a la coerción externa, es el hecho de sentir vergüenza uno de los modos de funcionamiento de la normatividad sexo genérica en el espacio universitario (Blanco, 2011, 2012a).

\section{Entrevista y construcción de conocimiento: voz y contramemoria}

Si la presentación del objeto de investigación constituyó una dificultad y un "escollo" inicial, una vez iniciada la entrevista en muchos casos se dio comienzo a un proceso de reconstrucción de la propia experiencia acerca del paso por la universidad que no había sido realizado antes. En la entrevista se produce una elaboración de una serie de acontecimientos que dan lugar a un relato en el que el punto de partida no es igual al punto de llegada, algo se produce en ese "forzar" un relato a partir del artificio de la entrevista y de una tecnología de la vida cotidiana como es la conversación. Así, los entrevistados han manifestado (durante o después de la entrevista) "saber más" o haber repensado cuestiones no antes problematizadas de sus propias biografías, que atañen a las regulaciones sexo genéricas en su experiencia como estudiantes. Para una entrevistada de Psicología (durante una segunda entrevista, habiendo ya leído la trascripción de la primera), el segundo encuentro constituyó un momento para reflexionar sobre aspectos antes no cuestionados de su 
activismo político universitario. En este sentido, hizo referencia a la persistencia de rasgos fuertes de la "dominación masculina", presentes en la dificultad para tomar la palabra en una asamblea ya que la voz del activismo universitario, aún entre quienes sostienen una agenda en torno al género y las mujeres, sigue estando hegemonizada por varones, o en las formas habilitadas para habitar el espacio público-político. Esta estudiante reflexiona:

En una época eran casi todos hombres los del Centro de Estudiantes. Presidentes, que yo recuerde, ya hace tres períodos consecutivos que son hombres. Del Partido Obrero: Manuel, Alejandro, Gabriel. Qué raro. Qué loco, ¿no? Son casi todas mujeres en la Facultad y los presidentes del Centro siempre son varones. Y la única que es muy pública es machona-machona. Se parece más a un hombre que a una chica, en su forma de manejarse, es increíble [...]. Las tres mujeres que tienen presencia son muy machonas. Y si no, son todos hombres. Los que pasan por los cursos son casi todos hombres ${ }^{10}$.

Esta reflexión tuvo lugar en la entrevista, y al momento no había encontrado lugar en los espacios de actividad habitual de la entrevistada: ni en la universidad como su lugar de aprendizaje científico-profesional, ni en esta como espacio de activismo político, ni de sociabilidad y vínculos de amistad y pareja.

A su vez, la entrevista constituye una instancia de autoría sobre la propia biografía. "Autoría" en el sentido -haciendo uso de la reflexión de Geertz (1989) - de que los entrevistados autorizan un relato sobre su propia experiencia. Identifico acá la posibilidad de emergencia tanto de un relato generacional, como de uno que permite desmontar aspectos del carácter heteronormativo de las narrativas en torno a la experiencia universitaria. Respecto del primer punto, vale señalar que las prácticas, representaciones y discursos de los jóvenes suelen ser leídas a partir de "esquemas pasados": los jóvenes del presente se piensan en términos de los jóvenes del pasado. En el caso de Argentina, el canon de interpretación de las prácticas y discursos de las juventudes (y con fuerza, respecto de los estudiantes universitarios) ha quedado marcado por la generación de la década de 1970, especialmente en torno a lo político (Chaves, 2005). En esta dirección, comprender los sentidos que adquieren las interseccionalidades entre "género y política" o "diversidad sexual y militancia estudiantil universitaria" desde la voz de los estudiantes en el presente permite atender a la convivencia de significaciones en disputa y que no se reduce al sentido que podían tener esos mismos cruces en otro ciclo histórico.

En segundo lugar, la voz de los entrevistados ha permitido acceder al relato de una experiencia de la vida en la universidad que pone en escena el carácter heteronormativo de esta institución. Si la heteronormatividad implica el espectro de relaciones sociales que se vuelven inteligibles a través de la heterosexualidad y que conllevan un sentido tácito de lo correcto y lo normal (Berlant y

10 Entrevista realizada el 5 de julio de 2010 a una estudiante que participa de una agrupación independiente en Psicología en un café del barrio de Flores cercano al lugar de trabajo de la entrevistada. 
Warner, 1999), hay aspectos de las biografías de quienes se reconocen alejados de esta expectativa común que no tienen las mismas posibilidades de inteligibilidad. En estos casos, las entrevistas pueden ser tomadas como una contramemoria respecto de la cultura estudiantil universitaria. Contramemoria, siguiendo a Henry Giroux atenta al lugar de los estudiantes y docentes como sujetos políticos, producida por la relectura del pasado a partir de las historias y voces específicas de los y las estudiantes, en tanto sujetos "que actúan dentro de configuraciones particulares de poder" (Giroux, 2003, 218) y en el que la narración de su experiencia universitaria, en el lenguaje de lo cotidiano, permite anudar esa singularidad con la vida pública y las tensiones permanentes entre el orden social y la diferencia. En este sentido, el trabajo sobre la voz estudiantil y el rescate de las particularidades de la vida universitaria a partir de las perspectivas de los sujetos, permite una comprensión de la institución "más allá" del movimiento estudiantil, como actor solapó en torno a los procesos colectivos de identificación, la heterogeneidad de lo político envueltos en la experiencia universitaria (Blanco, 2014).

\section{Palabras finales}

En este artículo se presentó la perspectiva de análisis, que articula distintos aportes en torno a lo que se denomina "el punto de vista del actor", y la estrategia metodológica cualitativa que orientó la investigación realizada entre los años 2007 y 2011. Esta tuvo por objeto analizar las regulaciones de las identidades de género y sexualidad en la vida cotidiana universitaria desde el punto de vista de los y las estudiantes, en un contexto de creciente tematización en la agenda pública de temas relativos al género y la sexualidad.

Se trabajó sobre el análisis de 36 entrevistas no directivas y de la observación participante y no participante en dos facultades de la UBA, Ciencias Exactas y Naturales, y Psicología. Como fue dicho, la elección de estas facultades se relaciona con la posibilidad de establecer comparaciones entre modos de atravesar la vida universitaria en instituciones con características diferentes, y constituyó a su vez un emergente del trabajo de campo ya que como acontecimientos singulares se identificó la existencia de un grupo de diversidad sexual en Ciencias Exactas y Naturales, y de grupos estudiantiles con una agenda en torno a "la mujer" y "el género" en Psicología.

La identificación de estos acontecimientos como significativos según las preguntas iniciales realizadas llevó a tomar una primera decisión. El procedimiento de indagación no se orientó hacia la descripción particularizada de estos fenómenos, sino de los vínculos de estos con el entramado más amplio en el que se inscriben y del que adquieren su sentido. En otras palabras, estos dos fenómenos importaban no en sus características intrínsecas sino como emergentes del entramado institucional y la cultura institucional en que se 
inscriben, especialmente en torno a las dimensiones de la vida cotidiana que configuraban la experiencia estudiantil en ambas facultades.

Las entrevistas tuvieron como objetivo entonces reconstruir la experiencia universitaria cotidiana a partir de las voces de "sujetos anónimos", y fueron realizadas no solo a quienes participaban de los grupos y agrupaciones de diversidad sexual, y en torno a la mujer y el género, sino también a un espectro heterogéneo de perfiles de jóvenes, muchos de estos menos imbuidos -a diferencia de quienes participan en grupos específicos- en una reflexividad acerca de la dimensión genérica y sexuada de la condición de estudiante. Por ello, como segunda decisión, las entrevistas fueron de carácter no directivo, con el propósito de que las respuestas se convirtieran en nuevas preguntas no estipuladas previamente. Esto implicó nuevos desafíos en torno al modo de presentar la investigación y de construir el propio lugar como investigador, que requirieron respuestas "más inmediatas que meditadas elaboraciones" y permitieron ir elaborando en situación aspectos de la estrategia que desarrollaba (Guber, 1991, 13). Es aquí donde los conceptos de reflexividad e implicación permiten repensar los cambios en la estrategia metodológica.

¿Cómo hablar de temas que pueden remitir al orden de lo íntimo para repensar una institución pública? El principal cambio en el curso del trabajo de campo refiere al abandono de una estrategia naturalista, ya que debido a los lazos "poco visibles" entre universidad y regulaciones sexo genéricas, esta resultaba inadecuada. El hecho de realizar un trabajo de indagación en un "universo próximo" (un universitario analizando las sociabilidades en el espacio universitario) me volvía objeto de reflexión para las propias personas entrevistadas quienes se interesaban por mi propia vinculación con la política, la militancia en la universidad, experiencia estudiantil e incluso identificación sexual. Esto redundó en una actividad constante, tensa y configurativa del trabajo de indagación que permitió la elaboración de nuevas preguntas.

A partir del cambio de estrategia, que habilitó una mayor confianza para desenvolver la indagación, en muchos casos se dio comienzo a un proceso de reconstrucción de la propia experiencia acerca del paso por la universidad que no había sido realizado antes. Esa elaboración permitió repensar cuestiones antes no problematizadas de sus propias biografías, que atañen a las regulaciones sexo genéricas en su experiencia como estudiantes. Así, la reflexividad que se produce en el momento de la entrevista, en ese relato en primera persona que realizan los y las estudiantes, habilita a una exploración del universo social y cultural universitario, y permite volver a narrar en el presente una institución que ya posee una historia sedimentada. Aquí se articula sincronía y diacronía, presente e historia, y sus yuxtaposiciones, lo que produce -en algunos casos- una relectura de la trayectoria biográfica pero también una reflexividad institucional: no es solo la propia historia sino también la historia institucional lo que se torna objeto de reflexión. Esta relectura del espacio universitario en el presente permite, por último, una revisión de los sentidos sedimentados de lo 
público. Si el orden de lo "público", de lo "privado" y de lo "íntimo" constituyen modos de ordenamiento de la experiencia que distribuyen en esferas aquello que requiere ocultarse y lo que necesita exhibirse públicamente para que exista (Arendt, 2011), la pregunta por las regulaciones sexo genérica en una institución poco explorada en torno a estas formas de normatividad social habilita un ejercicio de contramemoria sobre la universidad.

Desde este lugar, permite construir opacidad sobre una institución que se presenta como "transparente" (como fue dicho, los vínculos entre universidad y normatividad sexo genérica han pasado desapercibidos) y al mismo tiempo identificar en esta institución las articulaciones con aquello que ha sido excluido, ocultado o llamado a discreción de la esfera pública. En otras palabras, el ejercicio de contramemoria permite volver la mirada sobre una vieja institución pero desde nuevas interrogantes, identificando los procesos cotidianos de regulación de las expresiones e identidades de género y sexualidad que configuran la vida en común.

\section{Referencias}

Agamben, Giorgio. (2003). Homo sacer: el poder soberano y la nuda vida. Valencia: Pre-textos.

Aguilar, Paula; Bacci, Claudia; Fernández Cordero, Laura; Insausti, Joaquín; Peller, Mariela y Oberti, Alejandra. (2009). La sociología en el tocador: apuntes sobre una intervención estético-política. Ciencias Sociales, (74), pp. 20-22.

Arendt, Hannah. (2011). La condición humana. Buenos Aires: Paidós.

Arfuch, Leonor. (1992). La interioridad pública. Buenos Aires: Instituto de Investigaciones, Facultad de Ciencias Sociales, UBA.

Blanco, Rafael. (2014). Estudiantes, militantes, activistas. Nuevas agendas de las agrupaciones universitarias en torno al género y la diversidad sexual. Perfiles Educativos, XXXVI(144), 140-156.

Blanco, Rafael. (2012a). Neutralizar o encarnar la vergüenza. Sociabilidad estudiantil y regulaciones sexo genéricas en la Universidad. En: Figari, Carlos; Jones, Daniel y Barrón López, Sara. (Comps.). La producción de la sexualidad. Políticas y regulaciones sexuales en Argentina. Buenos Aires: Biblos.

Blanco, Rafael. (2012b). La dimensión sexuada de la experiencia estudiantil (Tesis doctoral). Universidad de Buenos Aires, Buenos Aires, Argentina.

Blanco, Rafael. (2011). Intimidad pública y experiencia estudiantil: regulaciones y subversión de las normas sexo-genéricas en la universidad. Revista de la Escuela de Ciencias de la Educación, 7(6), 87-100. 
Barthes, Roland. (2005). Cómo vivir juntos: simulaciones novelescas de algunos espacios cotidianos (notas de cursos y seminarios en el Collège de France, 1976-1977). Buenos Aires: Siglo XXI.

Berger, Peter y Luckmann, Thomas. (2006). La construcción social de la realidad. Buenos Aires: Amorrortu.

Berland, Lauren y Warner, Michael. (2002). Sexo en público. En Mérida, Rafael. (Ed.). Sexualidades transgresoras. Una antología de estudios queer. Barcelona: Icaria.

Bertaux, Daniel. (2005). Los relatos de vida: perspectiva etnosociológica. Barcelona: Bellaterra.

Bourdieu, Pierre. (1997). La ilusión biográfica. En Razones prácticas sobre la teoría de la acción. Barcelona: Anagrama.

Carli, Sandra. (2012). El estudiante universitario: hacia una historia del presente de la educación pública. Buenos Aires: Siglo XXI.

Coordinación General de Planificación Estratégica e Institucional. (2004). Censo de Estudiantes 2004. Buenos Aires: Universidad de Buenos Aires.

Coordinación General de Planificación Estratégica e Institucional. (2004). Censo docente 2004. Buenos Aires: Universidad de Buenos Aires.

Chaves, Mariana. (2005). Juventud negada y negativizada: Representaciones y formaciones discursivas vigentes en la Argentina contemporánea. Revista última Década, 13(23), 9-32.

Elías, Norbert. (2009). El proceso de la civilización. Buenos Aires: Fondo de Cultura Económica.

Facultad de Periodismo y Comunicación (UNLP). Recuperado de http://www. perio.unlp.edu.ar/node/2182

Feixa, Carles. (1998). De jóvenes, bandas y tribus: antropología de la juventud. Barcelona: Ariel.

Ford, Aníbal. (1996). Navegaciones: comunicación, cultura y crisis. Buenos Aires: Amorrortu.

Frederic, Sabina. (1998). Rehaciendo el campo: el lugar del etnógrafo entre el naturalismo y la reflexividad. Publicar en Antropología y Ciencias Sociales, (7), 85-103.

García Guadilla, Carmen. (2002). Tensiones y transiciones: educación superior latinoamericana en los albores del tercer milenio. Caracas: Nueva Sociedad. 
Geertz, Clifford. (1989). Estar allí. La antropología y la escena de la escritura. En El antropólogo como autor. Barcelona: Paidós.

Geertz, Clifford. (1994). "Desde el punto de vista del nativo": sobre la naturaleza del conocimiento antropológico. En Conocimiento local: ensayos sobre la interpretación de las culturas. Barcelona: Gedisa.

Giroux, Henry. (2003). Pedagogía y política de la esperanza. Teoría, cultura y enseñanza. Buenos Aires: Amorrortu.

Glaser, Barney y Strauss, Anselm. (1967). The discovery of Grounded Theory: Strategies of Qualitative Research. Chicago: Aldine.

Gogna, Mónica; Pecheny, Mario y Jones, Daniel. (2010). Enseñanza sobre género y sexualidad en universidades públicas en la Argentina. En Ortiz Ortega, Alicia y Pecheny, Mario. (Comps.). Enseñanza universitaria sobre género y sexualidades en Argentina, Chile, China, México y Sudáfrica. Buenos Aires: Teseo.

GTTCE (Grupo-Taller de Trabajo de Campo del Ides). (1999). De las notas de campo a la teoría: descubrimiento y redefinición de Nagual en los registros chiapanecos de Esther Hermitte. Antropología y Ciencias Sociales, VII-VIII, 69-92.

Guber, Rosana. (2001). La Etnografía: método, campo y reflexividad. Bogotá: Norma.

Guber, Rosana. (1991). El salvaje metropolitano: a la vuelta de la antropología postmoderna: reconstrucción del conocimiento social en el trabajo de campo. Buenos Aires: Legasa.

Hernández, Valeria. (2006). Estudiando el orden jerárquico a través del dispositivo implicación-reflexividad. Cuadernos de Antropología Social, (23), 57-80.

Humphreys, Laud. (1975). Tearoom trade: a study of homosexual encounters in public places. London: Duckworth.

Illouz, Eva. (2007). Intimidades Congeladas. Las emociones en el capitalismo. Buenos Aires: Katz.

Kosofsky-Sedwick, Eve. (1999). Performatividad queer: The art of the novel de Henry James. Revista Nómadas, (10), 198-214.

Lindón, Alicia. (Coord.). (2000). La vida cotidiana y su espacio-temporalidad. México: Anthropos.

Lourau, René. (1991). Implicación y sobreimplicación. En El espacio Institucional: la dimensión institucional de las prácticas sociales. Buenos Aires: Asociacion Civil El Espacio Institucional. 
Moreno, Aluminé. (2008). La invisibilidad como injusticia: estrategias del movimiento de la diversidad sexual. En Pecheny, Mario; Figari, Carlos y Jones, Daniel. (Comps.). Todo Sexo es Político: estudios sobre sexualidades en Argentina. Buenos Aires: El Zorzal.

Pecheny, Mario. (2008). Introducción: investigar sobre sujetos sexuales. En Pecheny, Mario; Figari, Carlos y Jones, Daniel. (Comps.). Todo Sexo es Político: estudios sobre sexualidades en Argentina. Buenos Aires: El Zorzal.

Pecheny, Mario. (2002). Identidades discretas. En Arfuch, Leonor. (Comp.). Identidades, sujetos y subjetividades. Buenos Aires: Prometeo.

Rockwell, Elsie. (2009). La experiencia etnográfica. Buenos Aires: Paidós.

Sautu, Ruth. (2004). Estilos y prácticas de la investigación biográfica. En Sautu, Ruth. (Comp.). El método biográfico: la reconstrucción de la sociedad a partir del testimonio de los actores. Buenos Aires: Lumiere. 